\title{
Qualidade da referenciação dos cuidados de saúde primários para a consulta de cefaleias de um hospital terciário
}

Ana Sofia Costa, ${ }^{1}$ Sara Varanda, ${ }^{2}$ Ana Filipa Santos, ${ }^{2}$ Susana Oliveira ${ }^{3}$

\section{RESUMO}

Introdução: As cefaleias são uma causa comum de procura dos cuidados de saúde primários, tendo reconhecido impacto na qualidade de vida.

Objetivo: Caracterizar a referenciação dos cuidados de saúde primários à consulta de cefaleias do serviço de neurologia de um hospital terciário, no norte de Portugal, e inferir àcerca da qualidade da sua informação.

Métodos: Estudo observacional, descritivo e transversal. A amostra compôs-se de doentes referenciados pelo médico de família para esta consulta, de outubro de 2014 a junho de 2018. Foram consultados os registos informáticos da referenciação. Categorizaram-se qualitativamente as referenciações, tendo em conta o número de variáveis descritas de um total de dez em estudo.

Resultados: A amostra compreendeu 144 doentes, com média de idades de 39 anos e predomínio de mulheres. Cerca de metade das referenciações não classificou a cefaleia. Das restantes, a maioria eram cefaleias primárias: enxaqueca não especificada $(29,2 \%)$, enxaqueca com aura $(7,6 \%)$ e cefaleia tipo tensão $(5,6 \%)$. Os motivos de referenciação mais frequentes foram a persistência das queixas e a refratariedade ao tratamento (19,4\%). Apesar disso, a maioria não mencionou o tratamento sintomático $(58,3 \%)$ e preventivo $(80,6 \%)$ realizado. O exame neurológico foi descrito em $23,6 \%$. A realização de exame de imagem foi relatada em $47,2 \%$. A qualidade das referenciações foi má em $18,8 \%$, insuficiente em $41,6 \%$, razoável em $32,0 \%$, boa em $6,9 \%$ e excelente em $0,7 \%$.

Conclusão: Este estudo encontrou lacunas importantes ao nível das referenciações, que podem ser otimizadas através de estratégias que aproximem os diferentes níveis de cuidados para melhorar a qualidade dos serviços.

Palavras-chave: Cefaleia; Cuidados de saúde primários; Referenciação e consulta.

\section{INTRODUÇÃO}

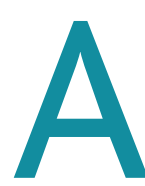
cefaleia é um sintoma que afeta pessoas de todas as idades a uma escala mundial..$^{1-2}$ Consiste numa das causas mais comuns de procura dos cuidados de saúde primários (CSP) e constitui um dos principais motivos de consulta do neurologista. ${ }^{2}$ Estima-se que cerca de $50 \%$ dos adultos apresentaram pelo menos um episódio de cefaleia no último ano. ${ }^{1}$ Contudo, sabe-se que a maioria não procura aju-

1. Médica Interna de Medicina Geral e Familiar. USF Gualtar, Braga.

2. Médica Assistente Hospitalar de Neurologia. Serviço de Neurologia, Hospital de Braga.

3. Médica Assistente Graduada de Medicina Geral e Familiar. USF Gualtar, Braga. da médica e faz a sua própria gestão destes episódios. ${ }^{1}$

A Classificação Internacional de Cefaleias (International Classification of Headache Disorders, $3^{\text {rd }}$ edition) divide as cefaleias em dois grandes grupos: as cefaleias primárias, que não têm etiologia demonstrável imagiológica e analiticamente e que são causadas por mecanismos patofisiológicos independentes, e as cefaleias secundárias, que apresentam uma causa conhecida que despoleta secundariamente a dor. ${ }^{3-4}$ As cefaleias primárias constituem de longe o tipo mais representativo, sendo a cefaleia tipo tensão e a enxaqueca as mais frequentes, com uma prevalência de $63 \%$ e $15 \%$, respetivamente. ${ }^{3}$ A cefaleia secundária mais comum ao ní- 
vel da consulta é a que ocorre por uso excessivo de medicamentos ( $1 \mathrm{a} 2 \%) .{ }^{3}$ Em aproximadamente $0,1 \%$ da população com cefaleias, este pode ser o sintoma de apresentação de patologias ameaçadoras da vida. ${ }^{5}$ Já a cefaleia crónica, definida pela presença de cefaleia em quinze ou mais dias por mês, durante pelo menos três meses, afeta 1,7 a $4 \%$ da população adulta mundial, podendo ser de etiologia primária ou secundária. ${ }^{1,3}$

Apesar do seu caráter maioritariamente benigno, as cefaleias constituem um problema de saúde pública devido ao impacto na qualidade de vida, à incapacidade que provocam e ao potencial uso excessivo de medicação, com as complicações neurológicas e sistémicas que daí advêm..$^{2-3} \mathrm{Na}$ medida em que a sua prevalência é maior em idade ativa, são responsáveis por elevado absentismo laboral e escolar e acarretam direta e indiretamente custos significativos à sociedade..$^{1,3}$ Quando cronificam podem associar-se a outras doenças, nomeadamente do foro depressivo e ansioso. ${ }^{1}$

Estudos internacionais mostraram que as cefaleias são responsáveis por 1,5 a 5,4\% de todas as consultas do médico de família, sendo que cerca de $2-3 \%$ desses doentes são referenciados para os cuidados de saúde secundários para avaliação mais especializada ${ }^{6} \mathrm{O}$ único estudo realizado em Portugal verificou que a cefaleia correspondeu a $0,7 \%$ do total de motivos de consulta e $2,1 \%$ do total de sinais/sintomas classificados pelo médico de família na International Classification for Primary Care - versão 2 (ICPC-2).?

A referenciação representa um veículo de informação singular entre o médico de família e o neurologista. Com este trabalho pretendeu-se caracterizar a referenciação dos CSP à consulta de cefaleias do serviço de neurologia de um hospital terciário, no norte de Portugal, e inferir acerca da qualidade da sua informação, de modo a identificar possíveis áreas de melhoria na articulação entre diferentes níveis dos cuidados de saúde.

\section{MÉTODOS}

Desenvolveu-se um estudo observacional, descritivo e transversal no serviço de neurologia de um hospital terciário.

O protocolo de investigação foi submetido à Comissão de Ética local e obteve parecer favorável.

$\mathrm{O}$ processo da referenciação à consulta hospitalar inicia-se no médico de família, com o envio da infor- mação clínica informaticamente, através da plataforma Alert ${ }^{\circledR} \mathrm{P} 1$. As referenciações são posteriormente avaliadas por um triador, que é um médico neurologista que direciona os doentes para a consulta de neurologia geral ou para a consulta de cefaleias, consoante a gravidade ou complexidade da cefaleia.

A amostra estudada correspondeu aos doentes referenciados pelos CSP para a consulta de neurologia e triados para a consulta de cefaleias, no período desde a criação desta consulta específica em outubro de 2014 até junho de 2018. Excluíram-se os doentes referenciados por outras especialidades hospitalares, por outras consultas de neurologia e pelo serviço de urgência. A amostra final incluiu 144 doentes.

Foram consultados os registos da referenciação feitos pelo médico de família, acessíveis através do registo médico eletrónico utilizado no referido hospital, para obter a informação necessária.

Das variáveis em estudo fizeram parte dados sociodemográficos, como a idade e o sexo, e dados relativos à referenciação, nomeadamente: motivo da referenciação, classificação da cefaleia, tempo de evolução, descrição e frequência da cefaleia, exame neurológico, presença de exame de imagem (tomografia computorizada ou ressonância magnética crânio-encefálica), utilização prévia de tratamento sintomático ou preventivo e existência de uso excessivo de medicamentos. As cefaleias foram classificadas de acordo com a terceira edição da Classificação Internacional de Cefaleias. ${ }^{4}$

A análise da referenciação feita pelo médico de família à consulta de cefaleias contemplou, assim, um total de dez variáveis. Tendo em conta o número de variáveis contido em cada referenciação, categorizou-se a qualidade das referenciações na seguinte escala qualitativa: má ( 1 a 2 variáveis descritas na referenciação), insuficiente (3 a 4 variáveis), razoável (5 a 6 variáveis), boa ( 7 a 8 variáveis) e excelente ( 9 a 10 variáveis).

Elaborou-se uma base de dados informática, devidamente codificada e sem qualquer dado identificativo dos participantes. Para a análise descritiva dos dados foram utilizados os programas informáticos Microsoft Excel ${ }^{\circledR}$ e SPSS ${ }^{\circledR}$ (Statistical Package for the Social Sciences), v. 20.0. As variáveis categóricas serão apresentadas como frequências e percentagens e as variáveis contínuas como médias e desvio-padrão para as variáveis que seguem distribuição normal. 


\begin{tabular}{|c|c|}
\hline $\begin{array}{l}\text { QUADRO I. Dados da referenciação: história clínic } \\
\text { objetivo }\end{array}$ & xame \\
\hline Motivo da referenciação & $n(\%)$ \\
\hline Não referido & $23(16)$ \\
\hline Cefaleia persistente & $28(19,4)$ \\
\hline Crises refratárias ao tratamento & $28(19,4)$ \\
\hline Aumento da frequência das crises & $19(13,2)$ \\
\hline Dúvida no diagnóstico & $17(11,8)$ \\
\hline Alteração encontrada em exame de imagem & $10(6,9)$ \\
\hline Sugestão do serviço de urgência ou de outro médico & $8(5,6)$ \\
\hline Atipia das características da cefaleia & $5(3,5)$ \\
\hline Alteração das características da cefaleia & $2(1,4)$ \\
\hline Intolerância à terapêutica & $2(1,4)$ \\
\hline Transferência de outro hospital por preferência & $2(1,4)$ \\
\hline Tempo de evolução & \\
\hline Não referido & $64(44,4)$ \\
\hline$<1$ ano & $47(32,6)$ \\
\hline 1 a 5 anos & $17(11,8)$ \\
\hline 5 a 10 anos & $3(2,1)$ \\
\hline$>10$ anos & $13(9,0)$ \\
\hline Classificação da cefaleia & \\
\hline Não referida & $71(49,3)$ \\
\hline Cefaleia primária & $66(45,8)$ \\
\hline Cefaleia tipo tensão & $8(5,5)$ \\
\hline Enxaqueca não especificada & $42(29,2)$ \\
\hline Enxaqueca sem aura & $1(0,7)$ \\
\hline Enxaqueca com aura & $11(7,6)$ \\
\hline Enxaqueca vestibular & $1(0,7)$ \\
\hline Cefaleia em salvas & $3(2,1)$ \\
\hline Cefaleia secundária & $3(2,1)$ \\
\hline Arterite & $2(1,4)$ \\
\hline Cefaleia pós-punção dural & $1(0,7)$ \\
\hline Neuropatias cranianas dolorosas e outras dores faciais & $4(2,8)$ \\
\hline Nevralgia & $4(2,8)$ \\
\hline Descrição da cefaleia & \\
\hline Não referida & $68(47,2)$ \\
\hline Referida & $76(52,8)$ \\
\hline Frequência da cefaleia & \\
\hline Não referida & $94(65,3)$ \\
\hline Referida & $50(34,7)$ \\
\hline Uso excessivo de medicamentos & \\
\hline Não referido & $144(100)$ \\
\hline Referido & $0(0)$ \\
\hline Exame neurológico & \\
\hline Não referido & $110(76,4)$ \\
\hline Referido & $34(23,6)$ \\
\hline
\end{tabular}

\section{RESULTADOS}

Desde que a consulta de cefaleias foi criada foram referenciados um total de 421 doentes, dos quais 144 provinham dos CSP e constituíram a amostra deste estudo.

Demograficamente, a idade dos doentes referenciados variou entre 9 e 84 anos, com média de 39,2 $( \pm 16,3)$ anos; a maioria era do sexo feminino $(73,6 \%)$.

Os dados da referenciação feita pelo médico de família, relativamente à história clínica e ao exame objetivo, encontram-se no Quadro I. Os motivos de referenciação mais reportados foram a existência de cefaleias persistentes $(19,4 \%)$ e de crises refratárias ao tratamento $(19,4 \%)$, seguindo-se o aumento da frequência das crises $(13,2 \%)$ e a dúvida no diagnóstico (11,8\%). Em 16\% não foi descrito o motivo do pedido desta consulta hospitalar. Quanto à classificação da cefaleia, a maioria foi categorizada como cefaleia primária, nomeadamente, enxaqueca não especificada $(29,2 \%)$, enxaqueca com aura $(7,6 \%)$ e cefaleia tipo tensão $(5,6 \%)$. Cerca de $2 \%$ eram cefaleias secundárias e $2,8 \%$ neuropatias cranianas dolorosas. Cerca de metade das referenciações não classificou a cefaleia (49,3\%). O tempo de evolução da cefaleia não foi referido em $44,4 \%$ dos doentes mas, quando referido, foi na maioria inferior a um ano. A descrição da cefaleia foi feita em $52,8 \%$ e a frequência das queixas em $34,7 \%$ das referenciações. O uso excessivo de medicamentos não foi reportado em nenhum dos doentes. Quanto aos dados do exame objetivo, $76,4 \%$ das referenciações não mencionaram ou descreveram o exame neurológico.

Até à data da referenciação, os triptanos foram o tratamento sintomático mais frequentemente referido (Quadro II). Em 3,5\% dos casos foi reportado o tratamento triplo com analgésico simples, anti-inflamatório não esteroide (AINE) e triptanos. Já os preventivos mais referidos em monoterapia foram o propranolol e o topiramato. Os tratamentos sintomático e preventivo realizados previamente não foram descritos em $58,3 \%$ e $80,5 \%$ das referenciações, respetivamente.

Relativamente ao estudo diagnóstico efetuado pelo médico de família verificou-se que $45,1 \%$ tinham realizado exame de imagem por tomografia 
computorizada e 2,1\% por ressonância magnética crânio-encefálica (Quadro III). Desses foi relatada alguma alteração imagiológica em 18 doentes (26,5\%).

A qualidade global das referenciações feitas pelos CSP para a consulta de cefaleias foi má em 18,8\%, insuficiente em $41,6 \%$ e razoável em $32 \%$ (Figura 1).

\section{DISCUSSÃO}

Os doentes referenciados à consulta de cefaleias apresentaram uma ampla faixa etária, desde a infância até à terceira idade, com uma média de idades no final da quarta década de vida e predomínio do sexo feminino (rácio feminino/masculino de 2,8/1), o que se assemelha à epidemiologia descrita na literatura. ${ }^{1-3}$

Quase metade das referenciações não classificaram o tipo de cefaleia, o que vai de encontro ao observado num estudo de coorte com médicos de família do Reino Unido, que constatou que $70 \%$ não estabeleciam um diagnóstico formal da cefaleia nas suas consultas. ${ }^{8}$ Por outro lado, a dúvida no diagnóstico também pode ser um dos motivos da baixa classificação das cefaleias, sendo que em $11,8 \%$ esse foi o motivo do pedido de consulta de neurologia.

Das cefaleias classificadas, a maioria enquadrou-se nas cefaleias primárias $(45,8 \%)$ e uma pequena percentagem nas secundárias $(2,1 \%)$ e nas neuropatias cranianas dolorosas $(2,8 \%)$, o que está de acordo com a literatura. ${ }^{1-3} \mathrm{~A}$ cefaleia primária mais prevalente nos doentes referenciados à consulta de neurologia foi a enxaqueca $(83,3 \%)$, tendo ultrapassado em grande margem a cefaleia tipo tensão $(12,1 \%)$. Apesar de a cefaleia tipo tensão ser a mais frequente nos estudos populacionais apresenta uma menor prevalência em níveis mais especializados de cuidados, muito possivelmente pelo seu menor impacto na qualidade de vida dos doentes..$^{1-3}$ Já a enxaqueca é um tipo de cefaleia incapacitante e que implica muitas vezes acompanhamento médico regular, podendo desse modo associar-se a maior dificuldade de gestão destes doentes e necessidade de orientação por neurologia. ${ }^{2}$ A cefaleia em salvas é uma das indicações para referenciação e avaliação pela especialidade de neurologia e foi o diagnóstico proposto em 2,1\% dos doentes. A cefaleia por uso excessivo de medicamentos não foi reportada em nenhuma referenciação, apesar de ser a cefaleia secundária mais prevalente na consulta e a causa mais co-

\begin{tabular}{|l|r|}
\hline $\begin{array}{l}|l| \\
\text { QUADRO II. Tratamentos sintomático e preventivo } \\
\text { prévios à referenciação }\end{array}$ \\
\hline Tratamento sintomático das cefaleias & $\mathbf{n}(\%)$ \\
\hline Não referido & $84(58,3)$ \\
Não medicados & $3(2,1)$ \\
Medicados & $57(39,6)$ \\
Triptanos & $20(13,8)$ \\
Analgésico simples & $8(5,5)$ \\
AINE & $7(4,9)$ \\
AINE + Triptano & $7(4,9)$ \\
Analgésico simples + AINE & $5(3,5)$ \\
Analgésico simples + AINE + Triptano & $5(3,5)$ \\
Opioides & $2(1,4)$ \\
Triptano + Opioide & $2(1,4)$ \\
Corticosteroide & $1(0,7)$ \\
\hline Tratamento preventivo das cefaleias & \\
\hline Não referido & $116(80,5)$ \\
Não medicados & $3(2,1)$ \\
Medicados & $25(17,4)$ \\
Propranolol & $7(4,9)$ \\
Topiramato & $7(4,9)$ \\
Amitriptilina & $5(3,5)$ \\
Propranolol + Topiramato & $4(2,7)$ \\
Carbamazepina & $1(0,7)$ \\
Propranolol + Topiramato + Valproato de sódio & $1(0,7)$ \\
\hline
\end{tabular}

QUADRO III. Exame de imagem a acompanhar a referenciação

\begin{tabular}{l|r} 
Exame de imagem & $\mathbf{n}(\%)$ \\
\hline Não referido & $76(52,8)$ \\
Referido & $68(47,2)$ \\
a) Tomografia computorizada crânio-encefálica & \\
$\quad$ Resultado normal & $50(34,7)$ \\
$\quad$ Alguma alteração relatada & $15(10,4)$ \\
b) Ressonância magnética crânio-encefálica & \\
$\quad$ Resultado normal & $0(0)$ \\
Alguma alteração relatada & $3(2,1)$
\end{tabular}

mum de transformação de uma cefaleia episódica numa cefaleia crónica, com uma prevalência na população com cefaleias de 1 a $2 \%$, pelo que poderá estar subdiagnosticada nesta amostra. ${ }^{1,3}$ 


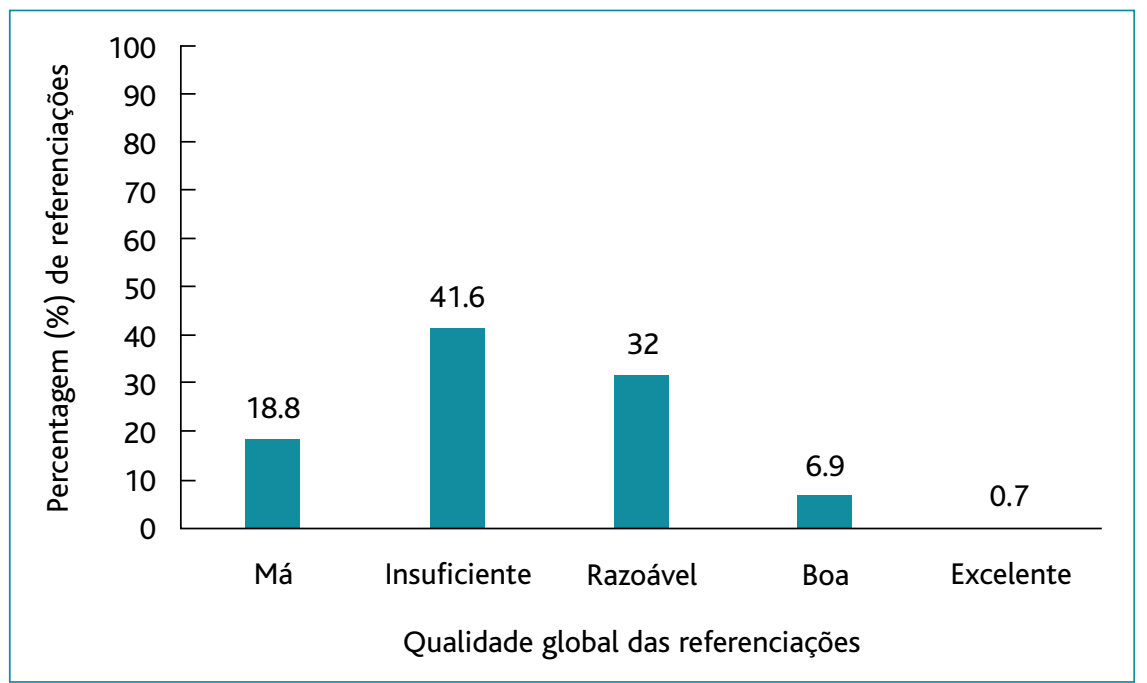

Figura 1. Qualidade global das referenciações dos CSP para a consulta de cefaleias. dos da América mais de 10 milhões de prescrições anualmente. ${ }^{9}$

Um dos fatores de gravidade da cefaleia e que, por vezes, faz suspeitar de uma cefaleia secundária é a presença de sinais neurológicos. Assim, a referência ao exame neurológico assume grande importância, sobretudo para a correta triagem hospitalar dos doentes. Neste estudo apenas $23,6 \%$ das referenciações incluíram esta informação.

Verificou-se ainda que $47,2 \%$ dos doentes tinha realizado exame de imagem previamente à referenciação, maioritariamente através
Os motivos de referenciação mais reportados foram a existência de cefaleias persistentes e de crises refratárias ao tratamento. Apesar disso, a maioria das referenciações não descreveu os tratamentos sintomático e/ou preventivo instituídos até à data do pedido de consulta hospitalar, o que constitui uma lacuna importante de informação clínica, que dificulta a avaliação da complexidade da cefaleia por parte do triador e a posterior orientação terapêutica na consulta de neurologia.

A maioria dos doentes em que o tempo de evolução foi mencionado na referenciação apresentava cefaleias há menos de um ano, mas em $9 \%$ dos casos era superior a dez anos. O presente estudo não tem dados suficientes para analisar se o impacto da cefaleia era maior nestes doentes com queixas de longa duração, mas sabe-se que o diagnóstico precoce e preciso é importante para a escolha do melhor tratamento para cada caso, de forma a limitar a incapacidade que a cefaleia pode provocar. ${ }^{2} \mathrm{~A}$ ausência de uma avaliação especializada precoce, quando necessária, pode ter sérias consequências, dada a possibilidade de evolução de uma cefaleia episódica para uma cefaleia crónica, como ocorre em cerca de $1,4 \%$ dos doentes com enxaqueca. ${ }^{9}$ Os doentes com enxaqueca crónica têm mais comorbilidades, maior utilização de recursos médicos, maior incapacidade e pior qualidade de vida do que os que sofrem de cefaleia episódica. ${ }^{9}$ Por outro lado, o uso de opioides nos indivíduos com dor crónica tem mostrado um perfil galopante, representando nos Estados Unide tomografia computorizada. A generalidade das cefaleias não apresenta indicação para a realização de estudo imagiológico; ${ }^{10}$ porém, é mais provável que os doentes enviados à consulta de cefaleias constituam casos de maior complexidade, o que justifica a sua mais frequente realização. Foram encontradas alterações imagiológicas em $26,5 \%$ dos casos que realizaram exame de imagem, muito embora a presença de pequenas alterações não seja na maioria dos casos patológica ou a causa da cefaleia.

A análise global das referenciações mostrou que apenas $6,9 \%$ e $0,7 \%$ tinham uma qualidade boa e excelente, respetivamente. Já $60,4 \%$ das referenciações apresentaram uma qualidade inferior ao que seria expectável (qualidade má e insuficiente). Estes números traduzem as lacunas de informação encontradas em grande parte das variáveis estudadas, que limitam a avaliação inicial da complexidade dos casos feita pelo triador a nível hospitalar e podem interferir no tempo de espera até à primeira consulta. Por outro lado, dificultam o acesso à informação clínica dos doentes por parte do neurologista, tornando a primeira avaliação na consulta morosa, com risco de instituição de planos terapêuticos que já haviam sido prescritos anteriormente pelo médico de família, podendo atrasar a resolução das queixas.

Este estudo apresenta algumas limitações, nomeadamente a caracterização retrospetiva das cefaleias e com base apenas nos registos da referenciação feita pe- 
los médicos de família, assim como o viés de triagem dos doentes, que poderá ter limitado a dimensão e o tipo da amostra. É relevante mencionar que este hospital terciário, até outubro de 2014, não tinha uma consulta específica de cefaleias nem um neurologista especializado nesta área; logo, o período de existência desta consulta é relativamente curto, podendo este constituir também um viés do estudo.

\section{CONCLUSÃO}

Através deste trabalho é possível concluir que as referenciações feitas pelos CSP à consulta de cefaleias apresentaram lacunas importantes na informação disponibilizada, que devem ser otimizadas. A referenciação dos doentes deve ser o mais completa possível para melhorar a triagem hospitalar, direcionando os casos mais complexos para a consulta específica de cefaleias e ajudando a priorizar a marcação das consultas. A referenciação é, de facto, um importante veículo de informação entre as diferentes especialidades, que subversivamente se pode tornar um obstáculo à orientação destes doentes.

A implementação de sessões de formação multidisciplinar dirigidas aos médicos de família, bem como a utilização das novas tecnologias para criação de plataformas educativas ou vídeos online, ${ }^{9}$ poderá contribuir para melhorar este panorama e sensibilizar os profissionais para esta temática. Por outro lado, seria também interessante utilizar as atuais plataformas informáticas em prol das necessidades reais do sistema de saúde, em particular a plataforma Alert ${ }^{\circledR} \mathrm{P} 1$, que poderia fomentar a uniformização da informação clínica através da presença de uma checklist dos dados fundamentais para a referenciação à consulta de cefaleias. Todas estas estratégias poderão aproximar os diferentes níveis de cuidados e, por conseguinte, aumentar a articulação e a qualidade dos cuidados de saúde prestados à população.

Não obstante, são necessários mais estudos para perceber se estes resultados são generalizáveis a outros locais do país e para investigar que dificuldades podem estar a ocorrer na prática clínica dos médicos de família que fundamentem estes resultados. Ao nível dos CSP, os tempos de consulta reduzidos, a alusão a múltiplas queixas pelo mesmo utente e a necessidade de gestão de vários problemas na mesma consulta, bem como a carga burocrática que assoberba diariamente os médicos de família, representam algumas das possíveis contrariedades ao estudo mais detalhado das cefaleias e à qualidade das referenciações.

\section{REFERÊNCIAS BIBLIOGRÁFICAS}

1. World Health Organization. Headache disorders [homepage]. Geneva: WHO; 2016 Apr 8 [ited 2018 Sep 15]. Available from: https://www.who. int/news-room/fact-sheets/detail/headache-disorders

2. Pedraza MI, Mulero P, Ruíz M, de la Cruz C, Herrero S, Guerrero AL. Characteristics of the first 2,000 patients registered in a specialist headache clinic. Neurologia. 2015;30(4):208-213.

3. Stovner LJ, Andree C. Prevalence of headache in Europe: a review for the Eurolight project. J Headache Pain. 2010;11(4):289-99.

4. Headache Classification Committee of the International Headache Society. The International Classification of Headache Disorders, 3rd edition. Cephalalgia. 2018;38(1):1-211.

5. Morgan $M$, Jenkins L, Ridsdale L. Patient pressure for referral for headache: a qualitative study of GPs' referral behaviour. $\mathrm{Br} J$ Gen Pract. 2007;57(534):29-35.

6. Frese T, Druckrey $H$, Sandholzer H. Headache in general practice: frequency, management, and results of encounter. Int Sch Res Notices. 2014;2014:169428.

7. Barreiro D, Santiago LM. Motivos de consulta em medicina geral e familiar no distrito de Coimbra no ano de 2010 [Reasons for clinical encounter in general practice/family medicine in 2010 in Central Portugal]. Rev Port Med Geral Fam. 2013;29(4):236-43. Portuguese

8. Kernick D, Stapley S, Hamilton W. GPs' classification of headache: is primary headache underdiagnosed? Br J Gen Pract. 2008;58(547):102-4.

9. Cowan R, Barad M. Effect of educating the primary care physician about headache to help reduce 'trivial' referrals and improve the number and quality of 'substantial' referrals that truly need subspecialty headache medicine care. Curr Treat Options Neurol. 2017;19(7):25.

10. Hainer BL, Matheson EM. Approach to acute headache in adults. Am Fam Physician. 2013;87(10):682-7.

\section{CONFLITO DE INTERESSES}

Os autores declaram não ter quaisquer conflitos de interesse.

\section{FINANCIAMENTO DO ESTUDO}

Os autores declaram que não receberam qualquer contributo financeiro adicional para a realização deste trabalho.

\section{ENDEREÇO PARA CORRESPONDÊNCIA \\ Ana Sofia Costa \\ E-mail: ana.s.dacostap@gmail.com \\ https://orcid.org/0000-0002-1340-2709}

Recebido em 01-08-2019

Aceite para publicação em 09-11-2019 


\section{ABSTRACT}

\section{QUALITY OF REFERRAL FROM PRIMARY HEALTH CARE TO A HEADACHE OUTPATIENT CLINIC IN A TERTIARY HOSPITAL}

Introduction: Headache is a common cause of primary health care consultation, and has a recognized impact on the quality of life.

Objectives: Characterize the primary health care referral to a headache outpatient clinic of a neurology department in a tertiary hospital in the north of Portugal and infer about information quality.

Methods: Observational, descriptive and transversal study. The sample included patients referred to headache consultation by their family doctors from October 2014 to June 2018. Referral records were consulted. The quality of the referral was qualitatively categorized taking into account the number of variables included, from a total of ten variables under study.

Results: The sample included 144 patients, with a mean age of 39 years, mostly women. Half of the referrals did not classify the headache.Among the others, the majority were primary headaches: unspecified migraine (29.2\%), migraine with aura (7.6\%) and tension headache $(5.6 \%)$. The most frequent reasons for referral were persistent complaints and treatment- refractory headache (19.4\%). Despite this, a large part did not mention symptomatic $(58.3 \%)$ and preventive $(80.6 \%)$ treatments attempted before. Neurological examination was described in $23.6 \%$. Imaging studies were reported in $47.2 \%$. The quality of referral information was poor in $18.8 \%$, insufficient in $41.6 \%$, reasonable in $32.0 \%$, good in $6.9 \%$ and excellent in $0.7 \%$.

Conclusion: This study found an important lack of information in referrals that can be optimized through strategies that bring different levels of care closer together to improve the quality of services.

Keywords: Headache; Primary health care; Referral and consultation. 